\title{
Author Correction: Mutational landscape and its clinical significance in paroxysmal nocturnal hemoglobinuria
}

Fangfei Chen, Shimin Hu(D, Jing Ruan, Miao Chen and Bing Han (1)

Correction to: Blood Cancer Journal

https://doi.org/10.1038/s41408-021-00451-1

published online 16 March 2021

When examining our submitted paper, we found 2 mistakes that need to be changed.

1. Author affiliations:

The affiliations of the first author Fangfei Chen should be

(1) Department of Hematology, Peking Union Medical College Hospital, Peking Union Medical College and
Chinese Academy of Medical Sciences, Beijing, China. (2) Division of Gastroenterology, The First Affiliated Hospital, Sun Yat-sen University, Guangzhou, China.

2. Funding:

One funding had been missed: The National Key Research and Development Program of China (2016YFC0901500).

Published online: 06 May 2021 